\title{
A general search for new phenomena at HERA and a search for magnetic monopoles
}

\author{
Ana Dubak* \\ On behalf of the $H 1$ collaboration \\ University of Montenegro / Max-Planck-Institute for Physics Munich \\ E-mail: dubak@mppmu .mpg • de
}

\begin{abstract}
This paper reports a model-independent general search for deviations from the Standard Model prediction and a search for magnetic monopoles in $e^{ \pm} p$ collisions at HERA. The general search is performed using $\mathrm{H} 1$ data taken in 1994-2000 corresponding to an integrated luminosity of $117 \mathrm{pb}^{-1}$. For the first time all event topologies involving isolated electrons, photons, muons, neutrinos and jets with high transverse momenta are investigated in a single analysis. Events are assigned to exclusive event classes according to their final state. A statistical algorithm is developed to search for deviations from the Standard Model in the distributions of the scalar sum of transverse momenta or invariant mass of final state particles. A good agreement with the Standard Model prediction is observed in most of the event classes. The most significant deviation is found for a topology containing an isolated muon, missing transverse momentum and a jet, consistent with a previously reported observation. The analysis is updated using data taken in 2004 corresponding to an integrated luminosity of $45 \mathrm{pb}^{-1}$. In addition, a direct search for magnetic monopoles produced in $e^{+} p$ collisions is presented. This search examines the beam pipe surrounding the H1 interaction region in 1995-1997. During this time an integrated luminosity of $62^{-1}$ was delivered. The beam pipe was investigated using a sensitive magnetometer to look for stopped magnetic monopoles. No magnetic monopoles were observed.
\end{abstract}

International Europhysics Conference on High Energy Physics

July 21st - 27th 2005

Lisboa, Portugal

* Speaker. 


\section{Introduction}

At HERA electrons and protons collide at a centre-of-mass energy of up to $319 \mathrm{GeV}$ providing a testing ground for the Standard Model complementary to $e^{+} e^{-}$and $p \bar{p}$ scattering. A general search for new physics has been performed by $\mathrm{H} 1$ collaboration for the first time in $e^{ \pm} p$ collisions using data from the full HERA I running period (1994-2000) taken at the centre-of-mass energies $\sqrt{s}=301,319 \mathrm{GeV}$ corresponding to an integrated luminosity of $118 \mathrm{pb}^{-1}$. This analysis is repeated using new data taken in 2004 at $\sqrt{s}=319 \mathrm{GeV}$ corresponding to a luminosity of $45 \mathrm{pb}^{-1}$.

Another outstanding issue in modern physics is the question of the existence of magnetic monopoles. Searches for magnetic monopoles produced in high energy particle collisions have been made in $p \bar{p}$ and $e^{+} e^{-}$interactions. No reproducible evidence has been found in any of them to support the existence of monopoles. The first direct search for magnetic monopoles produced in $e p$ collisions at $\sqrt{s}=301 \mathrm{GeV}$ is presented here. The monopoles are searched by examining the aluminum beam pipe which surrounded the H1 interaction point from 1995 to 1997 and was exposed to a luminosity of $62^{-1}$.

\section{General search for new phenomena}

The general search for new physics involving high $P_{T}$ particle production consists of a comprehensive and generic search for deviations from the SM prediction using all high $P_{T}$ final state

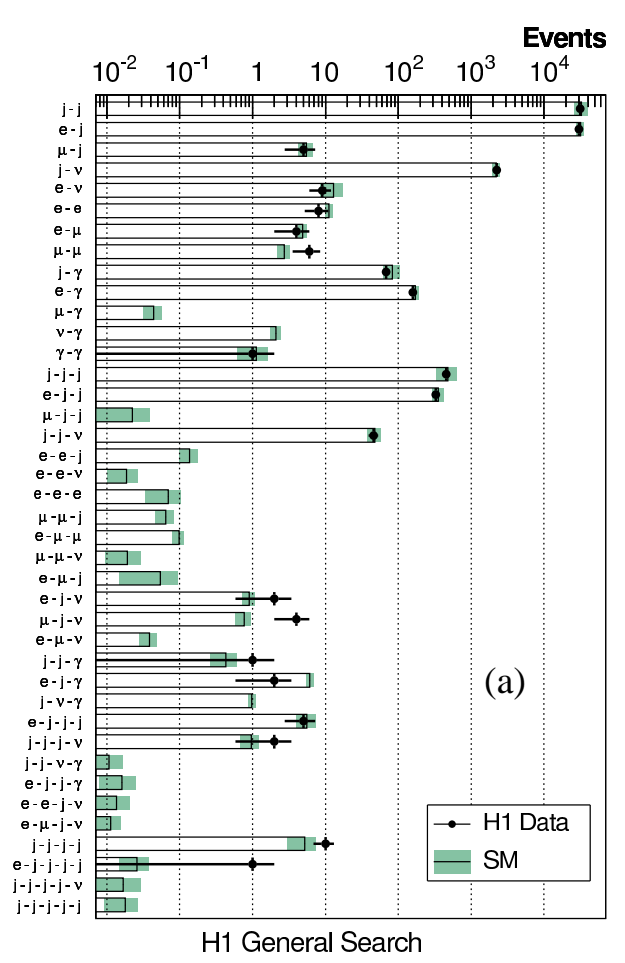

\section{Events}

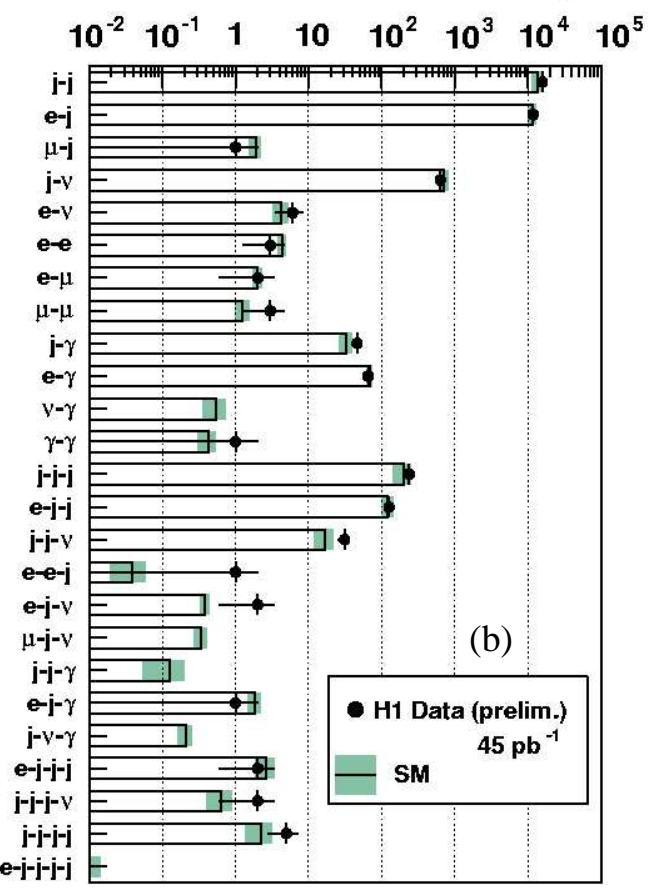

Figure 1: The data and the SM expectation for all event classes with a SM expectation greater than 0.01 events. The analysed data sample corresponds to an integrated luminosity of $117 \mathrm{pb}^{-1}$ (a) and $45 \mathrm{pb}^{-1}(\mathrm{~b})$ taken in 1994-2000 and 2004, respectively. 
configurations involving electrons $(e)$, muons $(\mu)$, jets $(j)$, photons $(\gamma)$ or neutrinos $(v)$ [1]. The analysis investigates all event classes which have at least two clearly identified and isolated objects with a minimum transverse momentum $P_{T}>20 \mathrm{GeV}$.

The event yields subdivided into event classes are presented for data and SM expectation in figure 1. All event classes with a SM expectation greater than 0.01 are shown. The other event classes contain no data events. Overall a good agreement of the SM prediction with the data is found. The largest deviation is observed in the $\mu-j-v$ event class which was previously reported [2].

In order to quantify the level of agreement between the data and the SM expectation and to identify regions of possible deviations, a search algorithm is developed (see [1] and [3]). This algorithm uses the observed and expected distributions of the invariant mass $\left(M_{\text {all }}\right)$ and the scalar sum of the transverse momentum $\left(\Sigma P_{T}\right)$ of the particles in the final state.

In a first step the algorithm searches for the region with the largest deviation in both, the $M_{\text {all }}$ and $\Sigma P_{T}$ spectra. For this all connected regions are checked and the probability $p$ that the SM expectation fluctuates to or above the observed number of data events in the region is calculated. The region of greatest deviation is the one with the smallest $p$-value, $p_{\min }$. To quantify the significance of the deviation per event class the probability $\hat{P}$ that a fluctuation with a $p$ value smaller than $p_{\text {min }}$ occurs anywhere in the distribution is calculated. The event class of most interest is the one with the smallest $\hat{P}$ value. The most significant deviation is found in the $\mu-j-v$ event class with $\hat{P}$ values of 0.010 and 0.001 for $M_{\text {all }}$ and $\Sigma P_{T}$, respectively. Finally, taking into account the large number of event classes the global probabilities to find at least one event class with a $\hat{P}$ value smaller than the observation in the $\mu-j-v$ channel are found to be $3 \%$ for the $\Sigma P_{T}$ and $28 \%$ for the $M_{\text {all }}$ distributions.

\section{Search for magnetic monopoles}

The direct search for magnetic monopoles is based on the fact that monopoles produced in very energetic $e^{+} p$ collision should fly away from the interaction region and be stopped in the material surrounding this region [4]. Trapped monopoles were hence searched for in the old aluminum beam pipe. The pipe was cut into long thin strips which were each passed through a superconducting

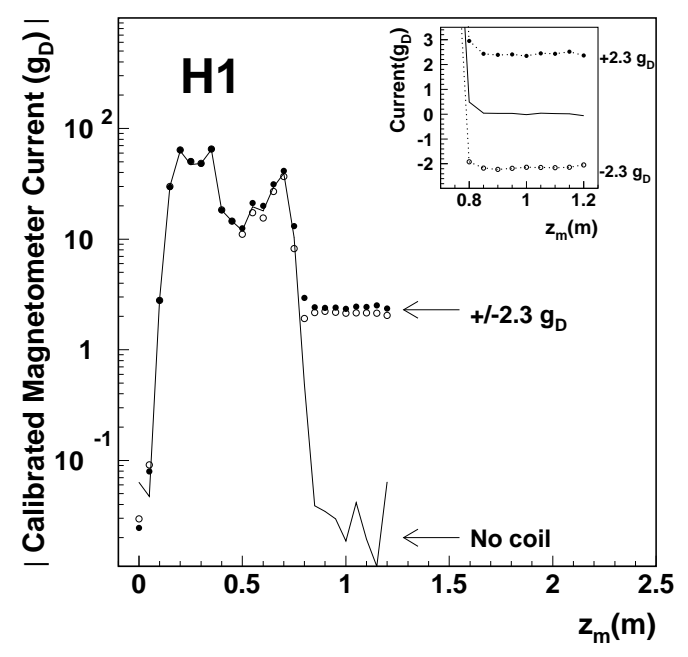

Figure 2: The absolute value of the calibrated magnetometer current on a logarithmic scale versus step position for a strip from the central beam pipe region. The solid line shows the measurements with the long strip alone. The closed (open) points show the measurements with a long strip together with a long calibration solenoid excited to simulate a monopole of strength $+2.3 g_{D}\left(-2.3 g_{D}\right)$. The inset shows the signed measurements of the calibrated magnetometer currents versus the step position on a linear scale. The expected persistent currents for monopoles of strength $\pm 2.3 g_{D}$ are shown by the arrow on the logarithmic plot and by the numbers in the margin on the inset linear plot. 
coil coupled to a magnetometer which was sensitive to monopoles of strength about 0.1 Dirac's monopole $\left(0.1 g_{D}\right)$.

Trapped magnetic monopoles in a strip would cause a current induced in the superconducting coil by the magnetic field of the monopole which persists after complete passage of the strip through the coil (see figure 2). In contrast, the induced currents from the magnetic fields of the permanent magnetic dipole moments in the material, equivalent to a series of equal and opposite magnetic charges, cancel so that the current due to dipoles returns to zero after passage of the strip.

All the strips from the central beam pipe, covering $0.3<z<0.3 \mathrm{~m}$, were passed through the magnetometer. The values of the residual persistent current are shown in figure 3. It can be seen that none of the fluctuations observed in single readings occured consistently in other readings on the same strip showing that no trapped monopole was present.

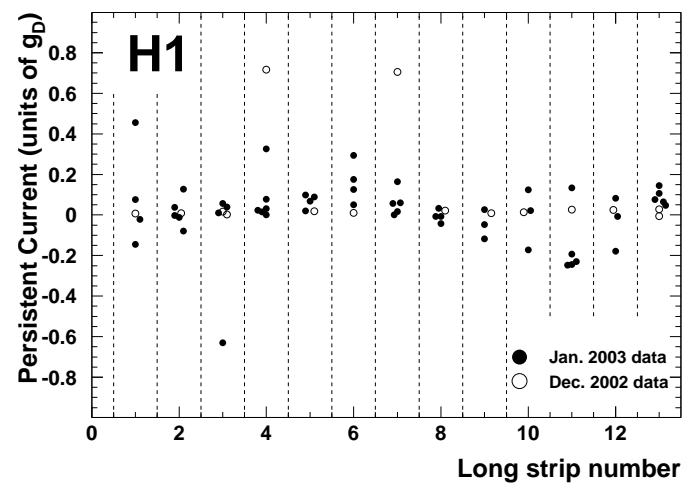

Figure 3: The measured persistent current in the long strips from the central beampipe in units of $g_{D}$, after passage through the magnetometer, against sample number. All strips were measured several times.

\section{Conclusion}

The data collected with the H1 experiment during the years 1994-2000 (HERA I) and 2004 (HERA II) have been investigated in a search for deviations from the SM prediction at high transverse momentum. For the first time all event topologies involving isolated electrons, photons, muons, neutrinos and jets are investigated in a single analysis. A good agreement between the data and the SM expectation is found in most event classes. The distributions in the invariant mass and scalar sum of transverse momenta of the particles in each event class have been systematically searched for deviations using a statistical algorithm. The most significant deviation is found in the $\mu-j-v$ event class, a topology where deviations have been previously reported.

A direct search for magnetic monopoles produced in $e^{+} p$ collisions at HERA has been made for the first time. Monopoles trapped in the beam pipe surrounding the interaction point were sought using a magnetometer which was sensitive down to 0.1 Dirac magnetic charges $\left(0.1 g_{D}\right)$. No monopole signal was observed.

\section{References}

[1] A. Aktas et al. [H1 Collaboration], Phys. Lett. B 602 (2004) 14, [arXiv:hep-ex/ 0408044 ].

[2] V. Andreev et al. [H1 Collaboration], Phys. Lett. B 561 (2003) 241, [arXiv: hep-ex/0301030].

[3] M. Wessels, Ph.D. Thesis, DESY-THESIS-2004-035.

[4] A. Aktas et al. [H1 Collaboration], Eur. Phys. J. C 41 (2005) 133 [arXiv: hep-ex/ 0501039 ]. 